\title{
A New High-Order Approximation for the Solution of Two-Space-Dimensional Quasilinear Hyperbolic Equations
}

\author{
R. K. Mohanty ${ }^{1}$ and Suruchi Singh ${ }^{1,2}$ \\ ${ }^{1}$ Department of Mathematics, Faculty of Mathematical Sciences, University of Delhi, Delhi 110 007, India \\ ${ }^{2}$ Department of Mathematics, Aditi Mahavidyalaya, University of Delhi, Delhi 110 039, India
}

Correspondence should be addressed to R. K. Mohanty, rmohanty@maths.du.ac.in

Received 31 March 2011; Revised 13 June 2011; Accepted 5 July 2011

Academic Editor: Ricardo Weder

Copyright (c) 2011 R. K. Mohanty and S. Singh. This is an open access article distributed under the Creative Commons Attribution License, which permits unrestricted use, distribution, and reproduction in any medium, provided the original work is properly cited.

we propose a new high-order approximation for the solution of two-space-dimensional quasilinear hyperbolic partial differential equation of the form $u_{t t}=A(x, y, t, u) u_{x x}+B(x, y, t, u) u_{y y}+g(x, y$, $\left.t, u, u_{x}, u_{y}, u_{t}\right), 0<x, y<1, t>0$ subject to appropriate initial and Dirichlet boundary conditions , where $k>0$ and $h>0$ are mesh sizes in time and space directions, respectively. We use only five evaluations of the function $g$ as compared to seven evaluations of the same function discussed by (Mohanty et al., 1996 and 2001). We describe the derivation procedure in details and also discuss how our formulation is able to handle the wave equation in polar coordinates. The proposed method when applied to a linear hyperbolic equation is also shown to be unconditionally stable. Some examples and their numerical results are provided to justify the usefulness of the proposed method.

\section{Introduction}

We consider the following two-space dimensional quasilinear hyperbolic partial differential equation:

$$
u_{t t}=A(x, y, t, u) u_{x x}+B(x, y, t, u) u_{y y}+g\left(x, y, t, u, u_{x}, u_{y}, u_{t}\right), \quad 0<x, y<1, t>0
$$

subject to the initial conditions

$$
u(x, y, 0)=\phi(x, y), \quad u_{t}(x, y, 0)=\psi(x, y), \quad 0 \leq x, y \leq 1
$$


and the boundary conditions

$$
\begin{gathered}
u(0, y, t)=a_{0}(y, t), \quad u(1, y, t)=a_{1}(y, t), \quad 0 \leq y \leq 1, t \geq 0, \\
u(x, 0, t)=b_{0}(x, t), \quad u(x, 1, t)=b_{1}(x, t), \quad 0 \leq x \leq 1, t \geq 0
\end{gathered}
$$

where (1.1) is assumed to satisfy the hyperbolicity condition $A(x, y, t, u)>0$ and $B(x, y, t, u)>0$ in the solution region $\Omega \equiv\{(x, y, t): 0<x, y<1, t>0\}$. Further we assume that $u(x, y, t) \in C^{6}, A(x, y, t, u), B(x, y, t, u) \in C^{4}$, and $\phi(x, y)$ and $\psi(x, y)$ are sufficiently differentiable function of as higher-order as possible.

Second-order quasilinear hyperbolic partial differential equation with appropriate initial and boundary conditions serves as models in many branches of physics and technology. Ciment and Leventhal $[1,2]$ have discussed operator compact implicit method to solve wave equation. Using finite volume technique, Schwartzkopff et al. [3] have studied high-order ADER schemes for first-order linear hyperbolic systems. Even much higher order ADER finite volume schemes have been presented and analyzed by Schwartzkopff et al. [4] on Cartesian meshes, and by Dumbser and Käser [5] on general two- and threedimensional unstructured meshes. In 1996, Mohanty et al. [6] have developed a highorder numerical method for the solution of two-space dimensional second-order nonlinear hyperbolic equation. Later, Mohanty et al. [7] have extended their technique to solve secondorder quasilinear hyperbolic equations. In both cases they have used seven evaluations of the function $g$ and 19-grid points. It has been shown that the linear schemes discussed in $[6,7]$ are conditionally stable. In the recent past, many researchers (see [8-16]) have developed unconditionally stable implicit finite difference methods for the solution of twospace dimensional linear hyperbolic equations with significant first derivative terms. Most recently, Mohanty and Singh [17] have derived a high accuracy numerical method based on Numerov type discretization for the solution of one space dimensional nonlinear hyperbolic equations, in which they have shown that the linear scheme is unconditionally stable.

In this paper, using nineteen grid-points, we derive a new compact three-level implicit numerical method of accuracy two in time and four in space for the solution of twospace dimensional quasilinear hyperbolic equation (1.1). In this method we require only five evaluations of the function $g$ as compared to seven evaluations of the same function discussed in $[6,7]$. In the next section, we give formulation of the method. In Section 3, we give the complete derivation of the method. In Section 4, we discuss the application of the proposed method to two-space dimensional wave equation in polar coordinates and discuss the stability analysis. In this section, we modify our method in such a way that the solution retains its order and accuracy everywhere in the vicinity of the singularity. In Section 5, we discuss superstable method for two-space dimensional telegraphic equation. In Section 6, we examine our new method over a set of linear and nonlinear second-order hyperbolic equations whose exact solutions are known and compared the results with the results of other known methods. Concluding remarks are given in Section 7.

\section{Formulation of the Numerical Method}

In this section, we aim to discuss a numerical method for the solution of nonlinear wave equation:

$$
u_{t t}=A(x, y, t) u_{x x}+B(x, y, t) u_{y y}+g\left(x, y, t, u, u_{x}, u_{y}, u_{t}\right), \quad 0<x, y<1, t>0 .
$$


Let $h>0$ and $k>0$ be the mesh spacing in the space and time directions, respectively. We replace the solution region $\Omega=\{(x, y, t) \mid 0<x, y<1, t>0\}$ by a set of grid points $\left(x_{l}, y_{m}, t_{j}\right)$, where $x_{l}=l h, l=0(1) N+1, y_{m}=m h, m=0(1) N+1$, and $t_{j}=j k, 0<j<J, N$ and $J$ being positive integers and $(N+1) h=1$. Let $p=k / h>0$ be the mesh ratio parameter. The exact values of $u(x, y, t), A(x, y, t)$, and $B(x, y, t)$ at the grid point $\left(x_{l}, y_{m}, t_{j}\right)$ are denoted by $U_{l, m^{\prime}}^{j} A_{l, m^{\prime}}^{j}$ and $B_{l, m^{\prime}}^{j}$, respectively. Similarly at the grid point $\left(x_{l}, y_{m}, t_{j}\right)$, we denote $A_{x_{l, m}}^{j}=$ $\partial A_{l, m}^{j} / \partial x, A_{y_{l, m}}^{j}=\partial A_{l, m}^{j} / \partial y, A_{x x_{l, m}}^{j}=\partial^{2} A_{l, m}^{j} / \partial x^{2}, \ldots$ and so forth. Let $u_{l, m}^{j}$ be the approximate value of $u(x, y, t)$ at the same grid point.

At the grid point $\left(x_{l}, y_{m}, t_{j}\right)$, we consider the following approximations:

$$
\begin{gathered}
\bar{U}_{t_{l, m}}^{j}=\frac{\left(U_{l, m}^{j+1}-U_{l, m}^{j-1}\right)}{(2 k)}, \\
\bar{U}_{t_{l \pm 1, m}}^{j}=\frac{\left(U_{l \pm 1, m}^{j+1}-U_{l \pm 1, m}^{j-1}\right)}{(2 k)}, \\
\bar{U}_{t_{l, m \pm 1}}^{j}=\frac{\left(U_{l, m \pm 1}^{j+1}-U_{l, m \pm 1}^{j-1}\right)}{(2 k)}, \\
\bar{U}_{t t_{l, m}}^{j}=\frac{\left(U_{l, m}^{j+1}-2 U_{l, m}^{j}+U_{l, m}^{j-1}\right)}{\left(k^{2}\right)}, \\
\bar{U}_{t t_{l \pm 1, m}}^{j}=\frac{\left(U_{l \pm 1, m}^{j+1}-2 U_{l \pm 1, m}^{j}+U_{l \pm 1, m}^{j-1}\right)}{\left(k^{2}\right)}, \\
\bar{U}_{t l_{l, m \pm 1}}^{j}=\frac{\left(U_{l, m \pm 1}^{j+1}-2 U_{l, m \pm 1}^{j}+U_{l, m \pm 1}^{j-1}\right)}{\left(k^{2}\right)}, \\
\bar{U}_{x x_{l, m}}^{j}=\frac{\left(U_{l+1, m}^{j}-2 U_{l, m}^{j}+U_{l-1, m}^{j}\right)}{\left(h^{2}\right)}, \\
\bar{U}_{x_{l \pm 1, m}}^{j}=\frac{\left(U_{l+1, m \pm 1}^{j}-2 U_{l, m \pm 1}^{j}+U_{l-1, m \pm 1}^{j}\right)}{\left(h^{2}\right)}, \\
\bar{U}_{x_{l, m}}^{j}=\frac{\left(U_{l+1, m}^{j}-U_{l-1, m}^{j}\right)}{(2 h)}, \\
(2 h)
\end{gathered}
$$




$$
\begin{gathered}
\bar{U}_{y_{l, m}}^{j}=\frac{\left(U_{l, m+1}^{j}-U_{l, m-1}^{j}\right)}{(2 h)}, \\
\bar{U}_{y_{l \pm 1, m}}^{j}=\frac{\left(U_{l \pm 1, m+1}^{j}-U_{l \pm 1, m-1}^{j}\right)}{(2 h)}, \\
\bar{U}_{y_{l, m \pm 1}}^{j}=\frac{\left( \pm 3 U_{l, m \pm 1}^{j} \mp 4 U_{l, m}^{j} \pm U_{l, m \mp 1}^{j}\right)}{(2 h)}, \\
\bar{U}_{y y_{l, m}}^{j}=\frac{\left(U_{l, m+1}^{j}-2 U_{l, m}^{j}+U_{l, m-1}^{j}\right)}{\left(h^{2}\right)}, \\
\bar{U}_{y y_{l \pm 1, m}}^{j}=\frac{\left(U_{l \pm 1, m+1}^{j}-2 U_{l \pm 1, m}^{j}+U_{l \pm 1, m-1}^{j}\right)}{\left(h^{2}\right)},
\end{gathered}
$$

$$
\begin{aligned}
& \bar{G}_{l \pm 1, m}^{j}=g\left(x_{l \pm 1}, y_{m}, t_{j}, U_{l \pm 1, m}^{j}, \bar{u}_{x_{l \pm 1, m}}^{j}, \bar{u}_{y_{l \pm 1, m}}^{j}, \bar{u}_{t_{l \pm 1, m}}^{j}\right), \\
& \bar{G}_{l, m \pm 1}^{j}=g\left(x_{l}, y_{m \pm 1}, t_{j}, U_{l, m \pm 1}^{j}, \bar{u}_{x_{l, m \pm 1}}^{j}, \bar{u}_{y_{l, m \pm 1}}^{j}, \bar{u}_{t_{l, m \pm 1}}^{j}\right),
\end{aligned}
$$

$$
\begin{aligned}
\overline{\bar{U}}_{x_{l, m}}^{j}= & \bar{U}_{x_{l, m}}^{j}+\frac{h}{16 A_{l, m}^{j}}\left(\bar{G}_{l+1, m}^{j}-\bar{G}_{l-1, m}^{j}\right)-\frac{h}{16 A_{l, m}^{j}}\left(\bar{U}_{t t_{l+1, m}}^{j}-\bar{U}_{t t_{l-1, m}}^{j}\right) \\
& +\frac{h B_{i, m}^{j}}{16 A_{l, m}^{j}}\left(\bar{U}_{y y_{l+1, m}}^{j}-\bar{U}_{y y_{l-1, m}}^{j}\right)+\frac{h^{2}}{8 A_{l, m}^{j}}\left(A_{x_{l, m}}^{j} \bar{U}_{x x_{l, m}}^{j}+B_{x_{l, m}}^{j} \bar{U}_{y y_{l, m}}^{j}\right),
\end{aligned}
$$$$
\overline{\bar{U}}_{y_{l, m}}^{j}=\bar{U}_{y_{l, m}}^{j}+\frac{h}{16 B_{l, m}^{j}}\left(\bar{G}_{l, m+1}^{j}-\bar{G}_{l, m-1}^{j}\right)-\frac{h}{16 B_{l, m}^{j}}\left(\bar{U}_{t t_{l, m+1}}^{j}-\bar{U}_{t l_{l, m-1}}^{j}\right)
$$$$
+\frac{h A_{l, m}^{j}}{16 B_{l, m}^{j}}\left(\bar{U}_{x x_{l, m+1}}^{j}-\bar{U}_{x x_{l, m-1}}^{j}\right)+\frac{h^{2}}{8 B_{l, m}^{j}}\left(A_{y_{l, m}}^{j} \bar{U}_{x x_{l, m}}^{j}+B_{y_{l, m}}^{j} \bar{U}_{y l_{l, m}}^{j}\right),
$$

$$
\overline{\bar{G}}_{l, m}^{j}=g\left(x_{l}, y_{m}, t_{j}, U_{l, m}^{j}, \overline{\bar{U}}_{x_{l, m}}^{j}, \overline{\bar{U}}_{y_{l, m}}^{j}, \bar{u}_{t_{l, m}}^{j}\right) .
$$


Advances in Mathematical Physics

Then at each grid point $\left(x_{l}, y_{m}, t_{j}\right), l, m=1,2, \ldots, N, j=2, \ldots, J$, an approximation with accuracy of $O\left(k^{2}+k^{2} h^{2}+h^{4}\right)$ for the solution of differential equation (2.1) may be written as

$$
\begin{aligned}
L_{u} \equiv & p^{2}\left[A_{l, m}^{j}-\frac{h^{2}}{6}\left(\frac{A_{x_{l, m}}^{j}}{A_{l, m}^{j}}\right) A_{x_{l, m}}^{j}-\frac{h^{2}}{6}\left(\frac{B_{y_{l, m}}^{j}}{B_{l, m}^{j}}\right) A_{y_{l, m}}^{j}+\frac{h^{2}}{12} A_{x x_{l, m}}^{j}+\frac{h^{2}}{12} A_{y y_{l, m}}^{j}\right] \delta_{x}^{2} U_{l, m}^{j} \\
+ & p^{2}\left[B_{l, m}^{j}-\frac{h^{2}}{6}\left(\frac{A_{x_{l, m}}^{j}}{A_{l, m}^{j}}\right) B_{x_{l, m}}^{j}-\frac{h^{2}}{6}\left(\frac{B_{y_{l, m}}^{j}}{B_{l, m}^{j}}\right) B_{y_{l, m}}^{j}+\frac{h^{2}}{12} B_{x x_{l, m}}^{j}+\frac{h^{2}}{12} B_{y y_{l, m}}^{j}\right] \delta_{y}^{2} U_{l, m}^{j} \\
+ & \frac{h p^{2}}{12}\left[A_{y_{l, m}}^{j}-\left(\frac{B_{y_{l, m}}^{j}}{B_{l, m}^{j}}\right) A_{l, m}^{j}\right]\left(\delta_{x}^{2} 2 \mu_{y} \delta_{y}\right) U_{l, m}^{j} \\
+ & \frac{h p^{2}}{12}\left[B_{x_{l, m}}^{j}-\left(\frac{A_{x_{l, m}}^{j}}{A_{l, m}^{j}}\right) B_{l, m}^{j}\right]\left(\delta_{y}^{2} 2 \mu_{x} \delta_{x}\right) U_{l, m}^{j}+\frac{p^{2}}{12}\left[A_{l, m}^{j}+B_{l, m}^{j}\right] \delta_{x}^{2} \delta_{y}^{2} U_{l, m}^{j} \\
= & \frac{k^{2}}{12}\left[\left(1-\frac{h A_{x_{l, m}}^{j}}{A_{l, m}^{j}}\right)^{j} \bar{U}_{t t_{l+1, m}}^{j}\left(1+\frac{h A_{x_{l, m}}^{j}}{A_{l, m}^{j}}\right) \bar{U}_{t t_{l-1, m}}^{j}+\left(1-\frac{h B_{y_{l, m}}^{j}}{B_{l, m}^{j}}\right) \bar{U}_{t t_{l, m+1}}^{j}\right. \\
& \left.+\left(1+\frac{h B_{y_{l, m}}^{j}}{B_{l, m}^{j}}\right) \bar{U}_{t t_{l, m-1}}^{j}+8 \bar{U}_{t l_{l, m}}^{j}\right] \\
+ & \frac{k^{2}}{12}\left[\left(1-\frac{h A_{x_{l, m}}^{j}}{A_{l, m}^{j}}\right)_{G_{l+1, m}^{j}}^{j}\left(1+\frac{h A_{x_{l, m}}^{j}}{A_{l, m}^{j}}\right) \bar{G}_{l-1, m}^{j}+\left(1-\frac{h B_{y_{l, m}}^{j}}{B_{l, m}^{j}}\right) \bar{G}_{l, m+1}^{j}\right. \\
& \left.+\left(1+\frac{h B_{y_{l, m}}^{j}}{B_{l, m}^{j}}\right) \bar{G}_{l, m-1}^{j}+8 \overline{\bar{G}}_{l, m}^{j}\right]+\bar{T}_{l, m}^{j}
\end{aligned}
$$

where $\delta_{x} U_{l, m}^{j}=\left(U_{l+\frac{1}{2}, m}^{j}-U_{l-\frac{1}{2}, m}^{j}\right), \mu_{x} U_{l, m}^{j}=(1 / 2)\left(U_{l+1 / 2, m}^{j}+U_{l-(1 / 2), m}^{j}\right), \ldots$ and so forth and $\bar{T}_{l, m}^{j}=O\left(k^{4}+k^{4} h^{2}+k^{2} h^{4}\right)$.

\section{Derivation Procedure of the Approximation}

At the grid point $\left(x_{l}, y_{m}, t_{j}\right)$, let us denote

$$
\alpha_{l, m}^{j}=\left(\frac{\partial g}{\partial U_{x}}\right)_{l, m}^{j}, \quad \beta_{l, m}^{j}=\left(\frac{\partial g}{\partial U_{y}}\right)_{l, m}^{j} .
$$


Further at the grid point $\left(x_{l}, y_{m}, t_{j}\right)$, the exact solution value $U_{l, m}^{j}$ satisfies

$$
\begin{aligned}
U_{t t_{l, m}}^{j} & -A\left(x_{l}, y_{m}, t_{j}\right) U_{x x_{l, m}}^{j}-B\left(x_{l}, y_{m}, t_{j}\right) U_{y y_{l, m}}^{j} \\
& =g\left(x_{l}, y_{m}, t_{j}, U_{l, m}^{j}, U_{l_{l, m}}^{j}, U_{y_{l, m}}^{j}, U_{l_{l, m}}^{j}\right) \equiv G_{l, m}^{j} \text { (say). }
\end{aligned}
$$

Using Taylor expansion about the grid point $\left(x_{l}, y_{m}, t_{j}\right)$, first we obtain

$$
\begin{aligned}
L_{u}=\frac{k^{2}}{12}[ & \left(1-\frac{h A_{x_{l, m}}^{j}}{A_{l, m}^{j}}\right) \bar{U}_{t t_{l+1, m}}^{j}+\left(1+\frac{h A_{x_{l, m}}^{j}}{A_{l, m}^{j}}\right) \bar{U}_{t t_{l-1, m}}^{j}+\left(1-\frac{h B_{l_{l, m}}^{j}}{B_{l, m}^{j}}\right) \bar{U}_{t t_{l, m+1}}^{j} \\
+ & \left.\left(1+\frac{h B_{y_{l, m}}^{j}}{B_{l, m}^{j}}\right) \bar{U}_{t t_{l, m-1}}^{j}+8 \bar{U}_{t t_{l, m}}^{j}\right] \\
-\frac{k^{2}}{12} & {\left[\left(1-\frac{h A_{l_{l, m}}^{j}}{A_{l, m}^{j}}\right) G_{l+1, m}^{j}+\left(1+\frac{h A_{x_{l, m}}^{j}}{A_{l, m}^{j}}\right) G_{l-1, m}^{j}+\left(1-\frac{h B_{y_{l, m}}^{j}}{B_{l, m}^{j}}\right) G_{l, m+1}^{j}\right.} \\
& \left.+\left(1+\frac{h B_{l_{l, m}}^{j}}{B_{l, m}^{j}}\right) G_{l, m-1}^{j}+8 G_{l, m}^{j}\right]+O\left(k^{4}+k^{4} h^{2}+k^{2} h^{4}\right) .
\end{aligned}
$$
obtain

With the help of the approximations (2.2a)-(2.2e), and from (2.5a) and (2.5b), we

$$
\begin{aligned}
& \bar{G}_{l \pm 1, m}^{j}=G_{l \pm 1, m}^{j}-\frac{h^{2}}{3} \frac{\partial^{3} U_{l, m}^{j}}{\partial x^{3}} \alpha_{l, m}^{j}+\frac{h^{2}}{6} \frac{\partial^{3} U_{l, m}^{j}}{\partial y^{3}} \beta_{l, m}^{j}+O\left(k^{2} \pm k^{2} h \pm h^{3}+h^{4}\right), \\
& \bar{G}_{l, m \pm 1}^{j}=G_{l, m \pm 1}^{j}+\frac{h^{2}}{6} \frac{\partial^{3} U_{l, m}^{j}}{\partial x^{3}} \alpha_{l, m}^{j}-\frac{h^{2}}{3} \frac{\partial^{3} U_{l, m}^{j}}{\partial y^{3}} \beta_{l, m}^{j}+O\left(k^{2} \pm k^{2} h \pm h^{3}+h^{4}\right) .
\end{aligned}
$$

Now we define the following approximations:

$$
\begin{aligned}
\overline{\bar{U}}_{x_{l, m}}^{j}= & \bar{U}_{x_{l, m}}^{j}+a_{11} h\left(\bar{G}_{l+1, m}^{j}-\bar{G}_{l-1, m}^{j}\right)+a_{12} h\left(\bar{U}_{t t_{l+1, m}}^{j}-\bar{U}_{t t_{l-1, m}}^{j}\right) \\
& +a_{13} h\left(\bar{U}_{y y_{l+1, m}}^{j}-\bar{U}_{y y_{l-1, m}}^{j}\right)+h^{2}\left(a_{14} \bar{U}_{x x_{l, m}}^{j}+a_{15} \bar{U}_{y l_{l, m}}^{j}\right), \\
\overline{\bar{U}}_{y_{l, m}}^{j}= & \bar{U}_{y_{l, m}}^{j}+b_{11} h\left(\bar{G}_{l, m+1}^{j}-\bar{G}_{l, m-1}^{j}\right)+b_{12} h\left(\bar{U}_{t t_{l, m+1}}^{j}-\bar{U}_{t t_{l, m-1}}^{j}\right) \\
& +b_{13} h\left(\bar{u}_{x x_{l, m+1}}^{j}-\bar{U}_{x x_{l, m-1}}^{j}\right)+h^{2}\left(b_{14} \bar{U}_{x x_{l, m}}^{j}+b_{15} \bar{u}_{y y_{l, m}}^{j}\right),
\end{aligned}
$$

where $a_{1 i}$ and $b_{1 i}, i=1,2, \ldots, 5$, are free parameters to be determined. 
By using the approximations (2.2a)-(2.4e) and (3.4), from (3.5), we get

$$
\begin{aligned}
& \overline{\bar{U}}_{x_{l, m}}^{j}=U_{x_{l, m}}^{j}+\frac{h^{2}}{6} T_{1}+O\left(k^{2}+k^{2} h^{2}+h^{4}\right), \\
& \overline{\bar{U}}_{y_{l, m}}^{j}=U_{y_{l, m}}^{j}+\frac{h^{2}}{6} T_{2}+O\left(k^{2}+k^{2} h^{2}+h^{4}\right),
\end{aligned}
$$

where

$$
\begin{aligned}
T_{1}= & \left(1-12 a_{11} A_{l, m}^{j}\right) U_{x x x_{l, m}}^{j}+12\left(a_{11}+a_{12}\right) U_{t t x_{l, m}}^{j}+12\left(-a_{11} B_{l, m}^{j}+a_{13}\right) U_{x y y_{l, m}}^{j} \\
& +\left(6 a_{14}-12 a_{11} A_{x_{l, m}}^{j}\right) U_{x x_{l, m}}^{j}+\left(6 a_{15}-12 a_{11} B_{x_{l, m}}^{j}\right) U_{y y_{l, m}{ }^{\prime}}^{j} \\
T_{2}= & \left(1-12 b_{11} B_{l, m}^{j}\right) U_{y y y_{l, m}}^{j}+12\left(b_{11}+b_{12}\right) U_{t t y_{l, m}}^{j}+12\left(-b_{11} A_{l, m}^{j}+b_{13}\right) U_{x x y_{l, m}}^{j} \\
& +\left(6 b_{14}-12 b_{11} A_{y_{l, m}}^{j}\right) U_{x x_{l, m}}^{j}+\left(6 b_{15}-12 b_{11} B_{y_{l, m}}^{j}\right) U_{y y_{l, m} .}^{j}
\end{aligned}
$$

Thus from (2.7), we obtain

$$
\overline{\bar{G}}_{l, m}^{j}=G_{l, m}^{j}+\frac{h^{2}}{6} T_{1} \alpha_{l, m}^{j}+\frac{h^{2}}{6} T_{2} \beta_{l, m}^{j}+O\left(k^{2}+k^{2} h^{2}+h^{4}\right) .
$$

Finally by the help of the approximations (3.4) and (3.8), from (2.8) and (3.3), we get

$$
\bar{T}_{l, m}^{j}=\frac{-k^{2} h^{2}}{36}\left(\frac{\partial^{3} U_{l, m}^{j}}{\partial x^{3}} \alpha_{l, m}^{j}+\frac{\partial^{3} U_{l, m}^{j}}{\partial y^{3}} \beta_{l, m}^{j}-4\left(T_{1} \alpha_{l, m}^{j}+T_{2} \beta_{l, m}^{j}\right)\right)+O\left(k^{4}+k^{4} h^{2}+k^{2} h^{4}\right) .
$$

In order to obtain the difference approximation of $O\left(k^{2}+k^{2} h^{2}+h^{4}\right)$ the coefficient of $k^{2} h^{2}$ in (3.9) must be zero, which gives the values of parameters:

$$
\begin{array}{cl}
a_{11}=\frac{1}{16 A_{l, m}^{j}}, \quad a_{12}=\frac{-1}{16 A_{l, m}^{j}}, \quad a_{13}=\frac{B_{l, m}^{j}}{16 A_{l, m}^{j}}, \quad a_{14}=\frac{A_{x_{l, m}}^{j}}{8 A_{l, m}^{j}}, & a_{15}=\frac{B_{l_{l, m}}^{j}}{8 A_{l, m}^{j}}, \\
b_{11}=\frac{1}{16 B_{l, m}^{j}}, & b_{12}=\frac{-1}{16 B_{l, m}^{j}}, \quad b_{13}=\frac{A_{l, m}^{j}}{16 B_{l, m}^{j}}, \quad b_{14}=\frac{A_{y_{l, m}}^{j}}{8 B_{l, m}^{j}}, \quad b_{15}=\frac{B_{y_{l, m}}^{j}}{8 B_{l, m}^{j}} .
\end{array}
$$

Thus we obtain the difference method of $O\left(k^{2}+k^{2} h^{2}+h^{4}\right)$ for the differential equation (2.1) and the local truncation error reduces to $\bar{T}_{l, m}^{j}=O\left(k^{4}+k^{4} h^{2}+k^{2} h^{4}\right)$.

Now we consider the numerical method of $O\left(k^{2}+k^{2} h^{2}+h^{4}\right)$ for the solution of the quasilinear hyperbolic equation (1.1). Whenever the coefficients are $A=A(x, y, t, u)$ and 
$B=B(x, y, t, u)$, the difference scheme (2.8) needs to be modified. For this purpose, we use the following central differences:

$$
\begin{gathered}
A_{x_{l, m}}^{j}=\frac{\left(A_{l+1, m}^{j}-A_{l-1, m}^{j}\right)}{(2 h)}+O\left(h^{2}\right), \\
A_{x x_{l, m}}^{j}=\frac{\left(A_{l+1, m}^{j}-2 A_{l, m}^{j}+A_{l-1, m}^{j}\right)}{\left(h^{2}\right)}+O\left(h^{2}\right), \\
A_{y_{l, m}}^{j}=\frac{\left(A_{l, m+1}^{j}-A_{l, m-1}^{j}\right)}{(2 h)}+O\left(h^{2}\right), \\
A_{y y_{l, m}}^{j}=\frac{\left(A_{l, m+1}^{j}-2 A_{l, m}^{j}+A_{l, m-1}^{j}\right)}{\left(h^{2}\right)}+O\left(h^{2}\right), \\
B_{x_{l, m}}^{j}=\frac{\left(B_{l+1, m}^{j}-B_{l-1, m}^{j}\right)}{(2 h)}+O\left(h^{2}\right), \\
B_{x x_{l, m}}^{j}=\frac{\left(B_{l+1, m}^{j}-2 B_{l, m}^{j}+B_{l-1, m}^{j}\right)}{\left(h^{2}\right)}+O\left(h^{2}\right), \\
B_{y_{l, m}}^{j}=\frac{\left(B_{l, m+1}^{j}-B_{l, m-1}^{j}\right)}{(2 h)}+O\left(h^{2}\right), \\
B_{y l_{l, m}}^{j}=\frac{\left(B_{l, m+1}^{j}-2 B_{l, m}^{j}+B_{l, m-1}^{j}\right)}{\left(h^{2}\right)}+O\left(h^{2}\right),
\end{gathered}
$$

where

$$
\begin{aligned}
& A_{l, m}^{j}=A\left(x_{l}, y_{m}, t_{j}, U_{l, m}^{j}\right), \quad A_{l \pm 1, m}^{j}=A\left(x_{l \pm 1}, y_{m}, t_{j}, U_{l \pm 1, m}^{j}\right), \quad A_{l, m \pm 1}^{j}=A\left(x_{l}, y_{m \pm 1}, t_{j}, U_{l, m \pm 1}^{j}\right), \\
& B_{l, m}^{j}=B\left(x_{l}, y_{m}, t_{j}, U_{l, m}^{j}\right), \quad B_{l \pm 1, m}^{j}=B\left(x_{l \pm 1}, y_{m}, t_{j}, U_{l \pm 1, m}^{j}\right), \quad B_{l, m \pm 1}^{j}=B\left(x_{l}, y_{m \pm 1}, t_{j}, U_{l, m \pm 1}^{j}\right) .
\end{aligned}
$$

By the help of the approximations (3.11), it is easy to verify that

$$
\begin{aligned}
A_{l, m}^{j}- & \frac{h^{2}}{6}\left(\frac{A_{x_{l, m}}^{j}}{A_{l, m}^{j}}\right) A_{x_{l, m}}^{j}-\frac{h^{2}}{6}\left(\frac{B_{y_{l, m}}^{j}}{B_{l, m}^{j}}\right) A_{y_{l, m}}^{j}+\frac{h^{2}}{12} A_{x x_{l, m}}^{j}+\frac{h^{2}}{12} A_{y y_{l, m}}^{j} \\
= & A_{l, m}^{j}-\frac{1}{24 A_{l, m}^{j}}\left(A_{l+1, m}^{j}-A_{l-1, m}^{j}\right)^{2}-\frac{1}{24 B_{l, m}^{j}}\left(B_{l, m+1}^{j}-B_{l, m-1}^{j}\right)\left(A_{l, m+1}^{j}-A_{l, m-1}^{j}\right) \\
& +\frac{1}{12}\left(A_{l+1, m}^{j}-2 A_{l, m}^{j}+A_{l-1, m}^{j}\right)+\frac{1}{12}\left(A_{l, m+1}^{j}-2 A_{l, m}^{j}+A_{l, m-1}^{j}\right)+O\left(h^{4}\right),
\end{aligned}
$$




$$
\begin{aligned}
B_{l, m}^{j}- & \frac{h^{2}}{6}\left(\frac{A_{x_{l, m}}^{j}}{A_{l, m}^{j}}\right) B_{x_{l, m}}^{j}-\frac{h^{2}}{6}\left(\frac{B_{y_{l, m}^{j}}^{j}}{B_{l, m}^{j}}\right) B_{y_{l, m}}^{j}+\frac{h^{2}}{12} B_{x x_{l, m}}^{j}+\frac{h^{2}}{12} B_{y y_{l, m}}^{j} \\
= & B_{l, m}^{j}-\frac{1}{24 B_{l, m}^{j}}\left(B_{l, m+1}^{j}-B_{l, m-1}^{j}\right)^{2}-\frac{1}{24 A_{l, m}^{j}}\left(A_{l+1, m}^{j}-A_{l-1, m}^{j}\right)\left(B_{l+1, m}^{j}-B_{l-1, m}^{j}\right) \\
& +\frac{1}{12}\left(B_{l+1, m}^{j}-2 B_{l, m}^{j}+B_{l-1, m}^{j}\right)+\frac{1}{12}\left(B_{l, m+1}^{j}-2 B_{l, m}^{j}+B_{l, m-1}^{j}\right)+O\left(h^{4}\right), \ldots, \text { and so forth. }
\end{aligned}
$$

Thus substituting the central difference approximations (3.11) into (2.8), we obtain the required numerical method of $O\left(k^{2}+k^{2} h^{2}+h^{4}\right)$ for the solution of quasilinear hyperbolic partial differential equation (1.1).

Note that the initial and Dirichlet boundary conditions are given by (1.2), (1.3a) and (1.3b), respectively. Incorporating the initial and boundary conditions, we can write the method (2.8) in a block tridiagonal matrix form. If the differential equation (1.1) is linear, we can solve the linear system using operator splitting method or, alternating direction implicit method (see $[18,19])$; in the nonlinear case, we can use Newton-Raphson iterative method to solve the non-linear system (see [20-24]).

\section{Stability Analysis}

In this section, we aim to discuss a stable difference scheme for the two-space dimensional linear hyperbolic equation with singular coefficients and ensure that the numerical method developed here retains its order and accuracy.

Let us consider the equation of the following form:

$$
u_{t t}=u_{x x}+u_{y y}+D(x) u_{x}+f(x, y, t), \quad 0<x, y<1, t>0
$$

Applying the approximation (2.8) to the differential equation (4.1), we obtain

$$
\begin{aligned}
p^{2}\left[\delta_{x}^{2}+\right. & \left.\delta_{y}^{2}+\frac{1}{6} \delta_{x}^{2} \delta_{y}^{2}\right] U_{l, m}^{j} \\
= & \frac{k^{2}}{12}\left[\bar{U}_{t t_{l+1, m}}^{j}+\bar{U}_{t t_{l-1, m}}^{j}+\bar{U}_{t t_{l, m+1}}^{j}+\bar{U}_{t t_{l, m-1}}^{j}+8 \overline{\bar{U}}_{t t_{l, m}}^{j}\right] \\
& -\frac{k^{2}}{12}\left[\bar{G}_{l+1, m}^{j}+\bar{G}_{l-1, m}^{j}+\bar{G}_{l, m+1}^{j}+\bar{G}_{l, m-1}^{j}+8 \overline{\bar{G}}_{l, m}^{j}\right]+O\left(k^{4}+k^{4} h^{2}+k^{2} h^{4}\right)
\end{aligned}
$$

$$
l=1,2, \ldots, N ; m=1,2, \ldots, N ; j=2,3, \ldots, J
$$


where we denote

$$
\begin{gathered}
D_{l}=D\left(x_{l}\right), \quad f_{l, m}^{j}=f\left(x_{l}, y_{m}, t_{j}\right), \\
\bar{G}_{l \pm 1, m}^{j}=D_{l \pm 1} \bar{U}_{x_{l \pm 1, m}}^{j}+f_{l \pm 1, m^{\prime}}^{j}, \\
\bar{G}_{l, m \pm 1}^{j}=D_{l} \bar{U}_{x_{l, m \pm 1}}^{j}+f_{l, m \pm 1^{\prime}}^{j} \\
\overline{\bar{G}}_{l, m}^{j}=D_{l} \overline{\bar{U}}_{x_{l, m}}^{j}+f_{l, m}^{j} .
\end{gathered}
$$

Note that the linear scheme (4.2) is of $O\left(k^{2}+k^{2} h^{2}+h^{4}\right)$ for the solution of differential equation (4.1). However, the scheme (4.2) fails to compute at $l=1$, if the coefficient $D(x)$ and/or the function $f(x, y, t)$ contains the singular terms like $1 / x, 1 / x^{2}, \ldots$, and so forth. We overcome this difficulty by modifying the method (4.2) in such a way that the solution retains its order and accuracy everywhere in the vicinity of singularity $x=0$.

We need the following approximations:

$$
\begin{gathered}
D_{l \pm 1}=D_{l} \pm h D_{x l}+\frac{h^{2}}{2} D_{x x l} \pm O\left(h^{3}\right), \\
f_{l \pm 1, m}^{j}=f_{l, m}^{j} \pm h f_{x_{l, m}}^{j}+\frac{h^{2}}{2} f_{x x_{l, m}}^{j} \pm O\left(h^{3}\right), \\
f_{l, m \pm 1}^{j}=f_{l, m}^{j} \pm h f_{y_{l, m}}^{j}+\frac{h^{2}}{2} f_{y y_{l, m}}^{j} \pm O\left(h^{3}\right) .
\end{gathered}
$$

Using the approximations (4.4) in the scheme (4.2) and neglecting local truncation error, we get

$$
\begin{aligned}
& {\left[1+\frac{1}{12}\left(\delta_{x}^{2}+R_{0}\left(2 \mu_{x} \delta_{x}\right)\right)+\frac{\delta_{y}^{2}}{12}\right] \delta_{t}^{2} u_{l, m}^{j}} \\
& \quad=p^{2}\left[R_{1} \delta_{x}^{2}+R_{2}\left(2 \mu_{x} \delta_{x}\right)+\delta_{y}^{2}+\frac{R_{0}}{6} \delta_{y}^{2}\left(2 \mu_{x} \delta_{x}\right)+\frac{1}{6} \delta_{x}^{2} \delta_{y}^{2}\right] u_{l, m}^{j}+\sum f,
\end{aligned}
$$

where

$$
\begin{gathered}
R_{0}=\frac{h D_{l}}{2}, \quad R_{1}=1+\frac{h^{2}}{12}\left(2 D_{x_{l}}+\left(D_{l}\right)^{2}\right), \quad R_{2}=R_{0}+\frac{h^{3}}{24}\left(D_{x x_{l}}+D_{l} D_{x_{l}}\right), \\
\sum f=\frac{k^{2}}{12}\left(12 f_{l, m}^{j}+h^{2}\left(f_{x x_{l, m}}^{j}+f_{y y_{l, m}}^{j}+D_{l} f_{x_{l, m}}^{j}\right)\right) .
\end{gathered}
$$


The previous scheme in product form may be written as

$$
\begin{aligned}
& {\left[1+\frac{1}{12}\left(\delta_{x}^{2}+R_{0}\left(2 \mu_{x} \delta_{x}\right)\right)\right]\left[1+\frac{\delta_{y}^{2}}{12}\right] \delta_{t}^{2} u_{l, m}^{j}} \\
& \quad=p^{2}\left[R_{1} \delta_{x}^{2}+R_{2}\left(2 \mu_{x} \delta_{x}\right)+\delta_{y}^{2}+\frac{R_{0}}{6} \delta_{y}^{2}\left(2 \mu_{x} \delta_{x}\right)+\frac{1}{6} \delta_{x}^{2} \delta_{y}^{2}\right] u_{l, m}^{j}+\sum f .
\end{aligned}
$$

It is difficult to find the stability region for the scheme (4.7). In order to obtain a valid stability region, we may modify the scheme (4.7) (see [23]) as

$$
\begin{aligned}
& {\left[1+\frac{1}{12}\left(R_{1} \delta_{x}^{2}+R_{2}\left(2 \mu_{x} \delta_{x}\right)\right)\right]\left[1+\frac{\delta_{y}^{2}}{12}\right] \delta_{t}^{2} u_{l, m}^{j}} \\
& \quad=p^{2}\left[R_{1} \delta_{x}^{2}+R_{2}\left(2 \mu_{x} \delta_{x}\right)+\delta_{y}^{2}+\frac{1}{6}\left(R_{1} \delta_{x}^{2}+R_{2}\left(2 \mu_{x} \delta_{x}\right)\right) \delta_{y}^{2}\right] u_{l, m}^{j}+\sum f .
\end{aligned}
$$

The additional terms added in (4.7) and (4.8) are of high orders and do not affect the accuracy of the scheme.

For stability, we put $u_{l, m}^{j}=A^{l} B^{m} \xi^{j} e^{i \beta l} e^{i r m}$ (where $\xi=e^{i \phi}$ such that $|\xi|=1$ ) in the homogeneous part of the scheme (4.8), and we get

$$
\xi-2+\xi^{-1}=-4 \sin ^{2} \frac{\phi}{2}=\frac{p^{2}\left[M_{1}+M_{2}+(1 / 6) M_{1} M_{2}\right]}{\left[1+(1 / 12) M_{1}\right]\left[1+(1 / 12) M_{2}\right]}
$$

where

$$
\begin{gathered}
M_{1}=R_{1}\left\{\left(A+A^{-1}\right) \cos \beta-2+i\left(A-A^{-1}\right) \sin \beta\right\}+R_{2}\left\{\left(A-A^{-1}\right) \cos \beta+i\left(A+A^{-1}\right) \sin \beta\right\}, \\
M_{2}=\left(B+B^{-1}\right) \cos \gamma-2+i\left(B-B^{-1}\right) \sin \gamma,
\end{gathered}
$$

where $A$ and $B$ are nonzero real parameters to be determined. Left-hand side of (4.9) is a real quantity. Thus the imaginary part of right-hand side of (4.9) must be zero. Thus we obtain

$$
\begin{gathered}
\left(R_{1}+R_{2}\right) A-\left(R_{1}-R_{2}\right) A^{-1}=0, \\
B-B^{-1}=0 .
\end{gathered}
$$

On solving, we get $A=\sqrt{\left(R_{1}-R_{2}\right) /\left(R_{1}+R_{2}\right)}, A^{-1}=\sqrt{\left(R_{1}+R_{2}\right) /\left(R_{1}-R_{2}\right)}$, and $B=B^{-1}=1$, provided that $R_{1} \pm R_{2}>0$,

Substituting the values of $A, A^{-1}, B$ and $B^{-1}$ in (4.10), we obtain

$$
M_{1}=-2\left[R_{1}+\sqrt{R_{1}^{2}-R_{2}^{2}}\left(2 \sin ^{2} \frac{\beta}{2}-1\right)\right], \quad M_{2}=-4 \sin ^{2} \frac{\gamma}{2} .
$$


Let $M_{1}=-2 M_{3}$ and $M_{2}=-4 M_{4}$, where

$$
M_{3}=R_{1}+\sqrt{R_{1}^{2}-R_{2}^{2}}\left(2 \sin ^{2} \frac{\beta}{2}-1\right), \quad M_{4}=\sin ^{2} \frac{\gamma}{2} .
$$

Substituting the values of $M_{1}$ and $M_{2}$ in (4.9), we obtain

$$
\sin ^{2} \frac{\phi}{2}=\frac{p^{2}\left[M_{3}+2 M_{4}-(2 / 3) M_{3} M_{4}\right]}{2\left[1-(1 / 6) M_{3}\right]\left[1-(1 / 3) M_{4}\right]}
$$

Since $\sin ^{2}(\phi / 2) \leq 1$, hence the method (4.8) is stable as long as

$$
\max \left(p^{2}\left[M_{3}+2 M_{4}-\frac{2}{3} M_{3} M_{4}\right]\right) \leq \min \left(2\left[1-\frac{1}{6} M_{3}\right]\left[1-\frac{1}{3} M_{4}\right]\right)
$$

this implies

$$
0<p^{2} \leq \frac{4-\left(R_{2}+\sqrt{{R_{2}{ }^{2}-R_{3}{ }^{2}}^{2}}\right)}{3\left(2+R_{2}+\sqrt{{R_{2}{ }^{2}-R_{3}^{2}}^{2}}\right)}
$$

which is the required stability interval for the scheme (4.8).

In order to facilitate the computation, we may rewrite (4.8) in operator split form (see $[18,19])$ as

$$
\begin{gathered}
{\left[1+\frac{\delta_{y}{ }^{2}}{12}\right] u_{l, m}^{*}=p^{2}\left[R_{1} \delta_{x}{ }^{2}+R_{2}\left(2 \mu_{x} \delta_{x}\right)+\delta_{y}{ }^{2}+\frac{1}{6}\left(R_{1} \delta_{x}{ }^{2}+R_{2}\left(2 \mu_{x} \delta_{x}\right)\right) \delta_{y}{ }^{2}\right] u_{l, m}^{j}+\sum f,} \\
{\left[1+\frac{1}{12}\left(R_{1} \delta_{x}{ }^{2}+R_{2}\left(2 \mu_{x} \delta_{x}\right)\right)\right] \delta_{t}{ }^{2} u_{l, m}^{j}=u_{l, m}^{*}}
\end{gathered}
$$

or,

$$
\left[1+\frac{1}{12}\left(R_{1} \delta_{x}^{2}+R_{2}\left(2 \mu_{x} \delta_{x}\right)\right)\right] u_{l, m}^{j+1}=u_{l, m}^{*}+\left[1+\frac{1}{12}\left(R_{1} \delta_{x}^{2}+R_{2}\left(2 \mu_{x} \delta_{x}\right)\right)\right]\left(2 u_{l, m}^{j}-u_{l, m}^{j-1}\right),
$$

where $u_{l, m}^{*}$ is an intermediate value. The intermediate boundary values required for solving (4.17) can be obtained from (4.18).

The left-hand sides of (4.17) and (4.18) are factorizations into $y$ - and $x$-differences, respectively, which allows us to solve by sweeping first (4.17) in the $y$ - and then (4.18) in the $x$-direction. It will be seen that these sweeps require only the solution of tri-diagonal systems. 


\section{Super Stable Method for Two-Dimensional Linear Hyperbolic Equation}

Let us consider the equation of the following form:

$$
u_{t t}+2 \alpha u_{t}+\beta^{2} u=u_{x x}+u_{y y}+f(x, y, t), \quad 0<x, y<1, t>0, \alpha>0, \beta \geq 0
$$

subject to appropriate initial and Dirichlet boundary conditions that are prescribed. The equation above represents two-space dimensional linear telegraphic hyperbolic equation. Applying the difference method (2.8) to the differential equation (5.1), we obtain

$$
\begin{aligned}
& \delta_{t}^{2} u_{l, m}^{j}+\sqrt{a}\left(2 \mu_{t} \delta_{t}\right) u_{l, m}^{j}+\frac{\sqrt{a}}{12}\left(\delta_{x}^{2}+\delta_{y}^{2}\right)\left(2 \mu_{t} \delta_{t}\right) u_{l, m}^{j}+b u_{l, m}^{j} \\
& \quad+\left(\frac{b}{12}-p^{2}\right)\left(\delta_{x}^{2}+\delta_{y}^{2}\right) u_{l, m}^{j}-\frac{p^{2}}{6} \delta_{x}^{2} \delta_{y}^{2} U_{l, m}^{j}+\frac{1}{12}\left(\delta_{x}^{2}+\delta_{y}^{2}\right) \delta_{t}^{2} U_{l, m}^{j}=F F,
\end{aligned}
$$

where

$$
a=\alpha^{2} k^{2}, \quad b=\beta^{2} k^{2}, \quad F F=\frac{k^{2}}{12}\left(f_{l+1, m}^{j}+f_{l-1, m}^{j}+f_{l, m+1}^{j}+f_{l, m-1}^{j}+8 f_{l, m}^{j}\right) .
$$

The previous scheme is conditionally stable (see [7]). In order to obtain a superstable method, we simply follow the ideas given by Chawla [24] and the scheme (5.2) may be rewritten as

$$
\begin{gathered}
{\left[1+\eta b^{2}+\left(\frac{1}{12}-r p^{2}\right)\left(\delta_{x}^{2}+\delta_{y}^{2}\right)\right] \delta_{t}^{2} u_{l, m}^{j}+\sqrt{a}\left(1+\frac{\delta_{x}^{2}}{12}+\frac{\delta_{y}^{2}}{12}\right)\left(2 \mu_{t} \delta_{t}\right) u_{l, m}^{j}} \\
=\left[\left(p^{2}-\frac{b}{12}\right)\left(\delta_{x}^{2}+\delta_{y}^{2}\right)+\frac{p^{2}}{6} \delta_{x}^{2} \delta_{y}^{2}-b\right] u_{l, m}^{j}+F F,
\end{gathered}
$$

where $\eta$ and $\gamma$ are free parameters to be determined. The additional terms are of high orders and do not affect the accuracy of the scheme.

To study stability for the scheme (5.4), we substitute $u_{l, m}^{j}=\xi^{j} e^{i \theta l} e^{i \phi m}$ in the homogeneous part of the (5.4), and we obtain the characteristic equation:

$$
A \xi^{2}+B \xi+C=0
$$


where

$$
\begin{aligned}
A= & 1+\eta b^{2}+\sqrt{a}-\frac{\sqrt{a}}{3}\left(\sin ^{2} \frac{\theta}{2}+\sin ^{2} \frac{\phi}{2}\right)+\left(4 \gamma p^{2}-\frac{1}{3}\right)\left(\sin ^{2} \frac{\theta}{2}+\sin ^{2} \frac{\phi}{2}\right), \\
B= & -2-2 \eta b^{2}+4\left(p^{2}-\frac{b}{12}\right)\left(\sin ^{2} \frac{\theta}{2}+\sin ^{2} \frac{\phi}{2}\right)+b \\
& +\left(\frac{2}{3}-8 \gamma p^{2}\right)\left(\sin ^{2} \frac{\theta}{2}+\sin ^{2} \frac{\phi}{2}\right)-\frac{8 p^{2}}{3} \sin ^{2} \frac{\theta}{2} \sin ^{2} \frac{\phi}{2}, \\
C= & 1+\eta b^{2}-\sqrt{a}+\frac{\sqrt{a}}{3}\left(\sin ^{2} \frac{\theta}{2}+\sin ^{2} \frac{\phi}{2}\right)+\left(4 \gamma p^{2}-\frac{1}{3}\right)\left(\sin ^{2} \frac{\theta}{2}+\sin ^{2} \frac{\phi}{2}\right) .
\end{aligned}
$$

Using the transformation $\xi=(1+z) /(1-z)$ in $(5.5)$, we obtain the transformed characteristic equation:

$$
(A-B+C) z^{2}+2(A-C) z+(A+B+C)=0 .
$$

For stability of the scheme (5.4), we must have the following conditions:

$$
A+B+C>0, \quad A-C>0, \quad A-B+C>0 .
$$

It is easy to verify that the coefficient is as follows:

$$
\begin{aligned}
A+B+C= & \frac{b}{6}\left(\sin ^{2} \frac{\theta}{2}+\sin ^{2} \frac{\phi}{2}\right)+\frac{b}{2}\left(\cos ^{2} \frac{\theta}{2}+\cos ^{2} \frac{\phi}{2}\right)+4 p^{2}\left(\sin ^{2} \frac{\theta}{2}+\sin ^{2} \frac{\phi}{2}-2 \sin ^{2} \frac{\theta}{2} \sin ^{2} \frac{\phi}{2}\right) \\
& +\frac{16 p^{2}}{3} \sin ^{2} \frac{\theta}{2} \sin ^{2} \frac{\phi}{2}>0 \text { for } \alpha>0, \beta \geq 0 \text {, and all } \theta, \phi \text {, except } \theta=\phi=0 \text { or } 2 \pi .
\end{aligned}
$$

We can treat this case separately.

The coefficient is as follows:

$$
A-C=\sqrt{a}\left(\frac{1}{3}\left(\sin ^{2} \frac{\theta}{2}+\sin ^{2} \frac{\phi}{2}\right)+\cos ^{2} \frac{\theta}{2}+\cos ^{2} \frac{\phi}{2}\right)>0 \text { for } \alpha>0, \quad \beta \geq 0, \text { and all } \theta, \phi
$$

For stability, it is required that the third coefficient is as follows:

$$
\begin{aligned}
A-B+C= & 4+4 \eta b^{2}-b+\frac{b}{3}\left(\sin ^{2} \frac{\theta}{2}+\sin ^{2} \frac{\phi}{2}\right) \\
& +4\left((4 \gamma-1) p^{2}-\frac{1}{3}\right)\left(\sin ^{2} \frac{\theta}{2}+\sin ^{2} \frac{\phi}{2}\right)+\frac{8 p^{2}}{3} \sin ^{2} \frac{\theta}{2} \sin ^{2} \frac{\phi}{2}>0 .
\end{aligned}
$$


Multiplying throughout of (5.11) by $16 \eta$, we get

$$
\begin{aligned}
& (64 \eta-1)+(8 \eta b-1)^{2}+\frac{16 b \eta}{3}\left(\sin ^{2} \frac{\theta}{2}+\sin ^{2} \frac{\phi}{2}\right) \\
& +64 \eta\left[(4 \gamma-1) p^{2}-\frac{1}{3}\right]\left(\sin ^{2} \frac{\theta}{2}+\sin ^{2} \frac{\phi}{2}\right)+\frac{128 p^{2}}{3} \sin ^{2} \frac{\theta}{2} \sin ^{2} \frac{\phi}{2}>0 .
\end{aligned}
$$

Thus the scheme is stable if

$$
\eta \geq \frac{1}{64}, \quad r \geq \frac{1+3 p^{2}}{12 p^{2}}
$$

For $\theta=\phi=0$ or $2 \pi$ and $\beta=0$, we have the characteristic equation:

$$
(1+\sqrt{a}) \xi^{2}-2 \xi+(1-\sqrt{a})=0
$$

whose roots are $\xi_{1,2}=1,(1-\sqrt{a}) /(1+\sqrt{a})=(1-\alpha k) /(1+\alpha k)$.

In this case also $|\xi| \leq 1$ and the scheme (5.4) is stable.

Hence for $\alpha>0, \beta \geq 0, \eta \geq 1 / 64, \gamma \geq\left(1+3 p^{2}\right) / 12 p^{2}$ (which are independent of $h$ and $k)$, the scheme (5.4) is superstable.

Further, the scheme (5.4) in product form may be written as

$$
\begin{aligned}
{[1+} & \left.\left(\frac{1}{12}-\gamma p^{2}\right) \delta_{y}^{2}\right]\left\{\left[1+\eta b^{2}+\left(\frac{1}{12}-\gamma p^{2}\right) \delta_{x}^{2}\right] \delta_{t}^{2} u_{l, m}^{j}+\sqrt{a}\left(1+\frac{\delta_{x}^{2}}{12}\right)\left(2 \mu_{t} \delta_{t}\right) u_{l, m}^{j}\right\} \\
& =\left[\left(p^{2}-\frac{b}{12}\right)\left(\delta_{x}^{2}+\delta_{y}^{2}\right)+\frac{p^{2}}{6} \delta_{x}^{2} \delta_{y}^{2}-b\right] u_{l, m}^{j}+F F .
\end{aligned}
$$

In this case also additional terms are of high orders, which do not affect the accuracy of the scheme. In order to facilitate the computation, we may rewrite the scheme (5.15) in two-step operator split form:

$$
\begin{gathered}
{\left[1+\left(\frac{1}{12}-r p^{2}\right) \delta_{y}^{2}\right] u_{l, m}^{*}=\left[\left(p^{2}-\frac{b}{12}\right)\left(\delta_{x}^{2}+\delta_{y}^{2}\right)+\frac{p^{2}}{6} \delta_{x}^{2} \delta_{y}^{2}-b\right] u_{l, m}^{j}+F F,} \\
{\left[1+\eta b^{2}+\left(\frac{1}{12}-r p^{2}\right) \delta_{x}^{2}\right] \delta_{t}^{2} u_{l, m}^{j}+\sqrt{a}\left(1+\frac{\delta_{x}^{2}}{12}\right)\left(2 \mu_{t} \delta_{t}\right) u_{l, m}^{j}=u_{l, m^{\prime}}^{*}}
\end{gathered}
$$

where $u_{l, m}^{*}$ is any intermediate value, and the intermediate boundary conditions required for the solution of $u_{l, m}^{*}$ may be obtained from (5.16b). The left-hand side matrices represented by (5.16a) and (5.16b) are tridiagonal, thus very easily solved in the region $0<x, y<1, t>0$ using a tridiagonal solver. 


\section{Numerical Illustrations}

In this section, we have solved some benchmark problems using the method described by (2.8) and compared our results with the results of the fourth-order numerical method discussed in [6, 7]. The exact solutions are provided in each case. The initial and boundary conditions may be obtained using the exact solution as a test procedure. The linear difference equations have been solved using a tridiagonal solver, whereas nonlinear difference equations have been solved using the Newton-Raphson method. While using the Newton-Raphson method, the iterations were stopped when absolute error tolerance $\leq 10^{-12}$ was achieved. In order to demonstrate the fourth-order convergence of the proposed method, for the computation of Example 6.1, we have chosen the fixed value of the parameter $\sigma=k / h^{2}=3.2$ for Example 6.1, and for other examples we have chosen the value of $\sigma=1.6$. For this choice, our method behaves like a fourth-order method in space. All computations were carried out using double precision arithmetic.

Note that the proposed method (2.8) for second-order hyperbolic equations is a threelevel scheme. The value of $u$ at $t=0$ is known from the initial condition. To start any computation, it is necessary to know the numerical value of $u$ of required accuracy at $t=k$. In this section, we discuss an explicit scheme of $O\left(k^{2}\right)$ for $u$ at first time level, that is, at $t=k$ in order to solve the differential equation (1.1) using the method (2.8), which is applicable to problems in both Cartesian and polar coordinates.

Since the values of $u$ and $u_{t}$ are known explicitly at $t=0$, this implies that all their successive tangential derivatives are known at $t=0$; that is, the values of $u, u_{x}, u_{x x}$, $u_{y}, u_{y y}, \ldots, u_{t}, u_{t x}, u_{t y}, \ldots$, and so forth are known at $t=0$.

An approximation for $u$ of $O\left(k^{2}\right)$ at $t=k$ may be written as

$$
u_{l, m}^{1}=u_{l, m}^{0}+k u_{t, m}^{0}+\frac{k^{2}}{2}\left(u_{t t}\right)_{l, m}^{0}+O\left(k^{3}\right)
$$

From (1.1), we have

$$
\left(u_{t t}\right)_{l, m}^{0}=\left[A(x, y, t, u) u_{x x}+B(x, y, t, u) u_{y y}+G\left(x, y, t, u, u_{x}, u_{y}, u_{t}\right)\right]_{l, m}^{0} .
$$

Thus using the initial values and their successive tangential derivative values, from (6.2) we can obtain the value of $\left(u_{t t}\right)_{l, m^{\prime}}^{0}$ and then ultimately, from (6.1) we can compute the value of $u$ at first time level, that is, at $t=k$.

Example 6.1 (the telegraphic equation with forcing function).

$$
u_{t t}+2 \alpha u_{t}+\beta^{2} u=u_{x x}+u_{y y}+\left(2-4 \alpha+\beta^{2}\right) e^{-2 t} \sinh x \sinh y, \quad 0<x, y<1, t>0,
$$

where $\alpha>0$ and $\beta \geq 0$ are real parameters. The exact solution is $u=e^{-2 t} \sinh x \sinh y$. The maximum absolute errors and CPU time (in seconds) are tabulated in Table 1 at $t=5$ for $\alpha>0$ and $\beta \geq 0$. 
Table 1: Example 6.1: the maximum absolute errors (using proposed $O\left(k^{2}+k^{2} h^{2}+h^{4}\right)$-method) (with CPU time).

\begin{tabular}{lccccc}
\hline \multirow{2}{*}{$\alpha=10, \beta=5}$, & $\alpha=20, \beta=10$, & $\alpha=40, \beta=4$, & $\alpha=50, \beta=5$, & $\alpha=10, \beta=0$, \\
& $\eta=0.5, \gamma=1$ & $\eta=1, \gamma=1$ & $\eta=10, \gamma=20$ & $\eta=0.25, \gamma=0.75$ & $\eta=5, \gamma=5$ \\
\hline \multirow{3}{*}{$1 / 8$ (CPU time) } & $0.5800 \mathrm{E}-07$ & $0.1784 \mathrm{E}-06$ & $0.4051 \mathrm{E}-02$ & $0.1119 \mathrm{E}-04$ & $0.6781 \mathrm{E}-04$ \\
& $(0.2087)$ & $(0.2781)$ & $(0.2084)$ & $(0.2214)$ & $(0.2120)$ \\
& $* 0.9234 \mathrm{E}-07$ & $* 0.6873 \mathrm{E}-06$ & $* 0.6677 \mathrm{E}-02$ & $* 0.8820 \mathrm{E}-04$ & $* 0.8873 \mathrm{E}-04$ \\
& $(0.2548)$ & $(0.3442)$ & $(0.2526)$ & $(0.2610)$ & $(0.2566)$ \\
\hline \multirow{3}{*}{$1 / 16$ (CPU time) } & $0.2390 \mathrm{E}-08$ & $0.2318 \mathrm{E}-08$ & $0.1930 \mathrm{E}-03$ & $0.6200 \mathrm{E}-06$ & $0.3125 \mathrm{E}-05$ \\
& $(1.9938)$ & $(2.0041)$ & $(1.9580)$ & $(1.9844)$ & $(1.9636)$ \\
& $* .6894 \mathrm{E}-08$ & $* 0.8329 \mathrm{E}-08$ & $* 0.3210 \mathrm{E}-03$ & $* 0.5033 \mathrm{E}-05$ & $* 0.5012 \mathrm{E}-05$ \\
& $(2.3690)$ & $(2.4715)$ & $(2.3427)$ & $(2.3677)$ & $(2.3525)$ \\
\hline & $0.1450 \mathrm{E}-09$ & $0.1132 \mathrm{E}-09$ & $0.1178 \mathrm{E}-04$ & $0.3899 \mathrm{E}-07$ & $0.1917 \mathrm{E}-06$ \\
$1 / 32$ (CPU time) & $(30.9160)$ & $(31.1875)$ & $(30.8334)$ & $(31.2224)$ & $(31.2644)$ \\
& $* 0.3634 \mathrm{E}-09$ & $* 0.4418 \mathrm{E}-09$ & $* 0.2144 \mathrm{E}-04$ & $* 0.2482 \mathrm{E}-06$ & $* 0.2898 \mathrm{E}-06$ \\
& $(38.8461)$ & $(39.1400)$ & $(38.3874)$ & $(39.3487)$ & $(39.9912)$ \\
\hline & $0.9055 \mathrm{E}-11$ & $0.6948 \mathrm{E}-11$ & $0.7365 \mathrm{E}-06$ & $0.2439 \mathrm{E}-08$ & $0.1198 \mathrm{E}-07$ \\
$1 / 64$ (CPU time) & $(493.5212)$ & $(503.4469)$ & $(492.8654)$ & $(498.1166)$ & $(500.2034)$ \\
& $* 0.2239 \mathrm{E}-10$ & $* 0.2746 \mathrm{E}-10$ & $* 0.1312 \mathrm{E}-05$ & $* 0.1419 \mathrm{E}-07$ & $* 0.1767 \mathrm{E}-07$ \\
& $(602.1290)$ & $(620.2021)$ & $(601.6682)$ & $(611.8769)$ & $(616.1248)$ \\
\hline
\end{tabular}

${ }^{*}$ Result obtained by using the Method discussed in [13].

Example 6.2 (wave equation in cylindrical polar coordinates).

$$
u_{t t}=u_{r r}+u_{z z}+\frac{1}{r} u_{r}-\left(3 \cosh r+\frac{\sinh r}{r}\right) \cosh z \sin t, \quad 0<r, z<1, t>0 .
$$

The aforementioned equation represents the two-space dimensional wave equation in cylindrical polar coordinates. The exact solution is $u=\cosh r \cosh z \sin t$. The maximum absolute errors and CPU time (in seconds) are tabulated in Table 2 at $t=1$ and $t=2$.

Example 6.3 (Van der Pol type nonlinear wave equation).

$$
\begin{array}{r}
u_{t t}=u_{x x}+u_{y y}+\gamma\left(u^{2}-1\right) u_{t}+\left(2 \pi^{2}+\gamma^{2} e^{-2 \gamma t} \sin ^{2}(\pi x) \sin ^{2}(\pi y)\right) e^{-\gamma t} \sin (\pi x) \sin (\pi y), \\
0<x, y<1, t>0,
\end{array}
$$

with exact solution $u=e^{-\gamma t} \sin (\pi x) \sin (\pi y)$. The maximum absolute errors and CPU time (in seconds) are tabulated in Table 3 at $t=2$ for $\gamma=1,2$, and 3 .

Example 6.4 (dissipative nonlinear wave equation).

$$
\begin{array}{r}
u_{t t}=u_{x x}+u_{y y}-2 u u_{t}+\left(2 \sin (\pi x) \sin (\pi y) \cos t+2 \pi^{2}-1\right) \sin (\pi x) \sin (\pi y) \sin t, \\
0<x, y<1, t>0,
\end{array}
$$


Table 2: Example 6.2: the maximum absolute errors (with CPU time).

\begin{tabular}{lcccc}
\hline$h$ & Proposed $O\left(k^{2}+k^{2} h^{2}+h^{4}\right)$-method & \multicolumn{2}{c}{$O\left(k^{4}+k^{2} h^{2}+h^{4}\right)$-method discussed in [6] } \\
& $t=1$ & $t=2$ & $t=1$ & $t=2$ \\
\hline \multirow{2}{*}{$1 / 8$ (CPU time) } & $0.1073 \mathrm{E}-05$ & $0.7883 \mathrm{E}-06$ & $0.8782 \mathrm{E}-05$ & $0.7260 \mathrm{E}-05$ \\
& $(0.1077)$ & $(0.1488)$ & $(0.1344)$ & $(0.1985)$ \\
$1 / 16$ (CPU time) & $0.6026 \mathrm{E}-07$ & $0.5424 \mathrm{E}-07$ & $0.4888 \mathrm{E}-06$ & $0.3842 \mathrm{E}-06$ \\
& $(0.6614)$ & $(1.2736)$ & $(1.0404)$ & $(1.6892)$ \\
$1 / 32$ (CPU time) & $0.4626 \mathrm{E}-08$ & $0.4533 \mathrm{E}-08$ & $0.2718 \mathrm{E}-07$ & $0.2146 \mathrm{E}-07$ \\
& $(9.2670)$ & $(16.4166)$ & $(12.8822)$ & $(20.3392)$ \\
$1 / 64$ (CPU time) & $0.2892 \mathrm{E}-09$ & $0.2864 \mathrm{E}-09$ & $0.1616 \mathrm{E}-08$ & $0.1284 \mathrm{E}-08$ \\
& $(142.9786)$ & $(300.5124)$ & $(178.3944)$ & $(380.6638)$ \\
\hline
\end{tabular}

Table 3: Example 6.3: the maximum absolute errors (with CPU time).

\begin{tabular}{lcccccc}
\hline \multirow{2}{*}{$h$} & \multicolumn{2}{c}{ Proposed $O\left(k^{2}+k^{2} h^{2}+h^{4}\right)$-method } & \multicolumn{4}{c}{$O\left(k^{4}+k^{2} h^{2}+h^{4}\right)$-method discussed in [6] } \\
& $\gamma=1$ & $r=2$ & $r=3$ & $r=1$ & $\gamma=2$ & $r=3$ \\
\hline \multirow{2}{*}{$1 / 4$ (CPU time) } & $0.4719 \mathrm{E}-03$ & $0.9207 \mathrm{E}-04$ & $0.2974 \mathrm{E}-04$ & $0.8616 \mathrm{E}-03$ & $0.7412 \mathrm{E}-03$ & $0.4433 \mathrm{E}-04$ \\
& $(0.2680)$ & $(0.2530)$ & $(0.2712)$ & $(0.3322)$ & $(0.3221)$ & $(0.3476)$ \\
$1 / 8$ (CPU time) & $0.3186 \mathrm{E}-04$ & $0.7369 \mathrm{E}-05$ & $0.2594 \mathrm{E}-06$ & $0.5050 \mathrm{E}-04$ & $0.4244 \mathrm{E}-04$ & $0.2211 \mathrm{E}-05$ \\
& $(3.0112)$ & $(2.9443)$ & $(2.9417)$ & $(3.8616)$ & $(3.7824)$ & $(3.8806)$ \\
$1 / 16$ (CPU time) & $0.2041 \mathrm{E}-05$ & $0.4868 \mathrm{E}-06$ & $0.1008 \mathrm{E}-07$ & $0.3368 \mathrm{E}-05$ & $0.2018 \mathrm{E}-05$ & $0.1060 \mathrm{E}-06$ \\
& $(49.9442)$ & $(47.6788)$ & $(49.0598)$ & $(63.8846)$ & $(62.1258)$ & $(63.1625)$ \\
$1 / 32$ (CPU time) & $0.1279 \mathrm{E}-06$ & $0.3061 \mathrm{E}-07$ & $0.6260 \mathrm{E}-09$ & $0.2100 \mathrm{E}-06$ & $0.1212 \mathrm{E}-06$ & $0.6214 \mathrm{E}-08$ \\
& $(703.2124)$ & $(659.7450)$ & $(685.7455)$ & $(879.6670)$ & $(825.1212)$ & $(832.6086)$ \\
\hline
\end{tabular}

with exact solution $u=\sin (\pi x) \sin (\pi y) \sin t$. The maximum absolute errors and CPU time (in seconds) are tabulated in Table 4 at $t=1$.

Example 6.5 (nonlinear wave equation with variable coefficients).

$$
\begin{aligned}
& u_{t t}=\left(1+x^{2}\right) u_{x x}+\left(1+y^{2}\right) u_{y y}+\gamma u\left(u_{x}+u_{y}+u_{t}\right) \\
&+ {\left[\gamma e^{-t}(\cosh x \cosh y-\sinh (x+y))-1-x^{2}-y^{2}\right] e^{-t} \cosh x \cosh y, } \\
& 0<x, y<1, t>0,
\end{aligned}
$$

with exact solution $u=e^{-t} \cosh x \cosh y$. The maximum absolute errors and CPU time (in seconds) are tabulated in Table 5 at $t=1$ for $\gamma=1,2$ and 5 . 
Table 4: Example 6.4: the maximum absolute errors (with CPU time).

\begin{tabular}{lcc}
\hline$h$ & Proposed $O\left(k^{2}+k^{2} h^{2}+h^{4}\right)$-method & $O\left(k^{4}+k^{2} h^{2}+h^{4}\right)$-method discussed in [6] \\
\hline $1 / 4$ (CPU time) & $0.8996 \mathrm{E}-03$ & $0.1440 \mathrm{E}-03$ \\
& $(0.1841)$ & $(0.2312)$ \\
$1 / 8$ (CPU time) & $0.5807 \mathrm{E}-04$ & $0.8614 \mathrm{E}-04$ \\
& $(1.4538)$ & $(1.8412)$ \\
$1 / 16$ (CPU time) & $0.3656 \mathrm{E}-05$ & $0.5644 \mathrm{E}-05$ \\
& $(22.3786)$ & $(27.1590)$ \\
$1 / 32$ (CPU time) & $0.2299 \mathrm{E}-06$ & $0.3246 \mathrm{E}-06$ \\
& $(307.0478)$ & $(384.4488)$ \\
\hline
\end{tabular}

Table 5: Example 6.5: The maximum absolute errors (with CPU time).

\begin{tabular}{lcccccc}
\hline \multirow{2}{*}{$h$} & \multicolumn{2}{c}{ Proposed $O\left(k^{2}+k^{2} h^{2}+h^{4}\right)$-method } & \multicolumn{4}{c}{$O\left(k^{4}+k^{2} h^{2}+h^{4}\right)$-method discussed in [6] } \\
& $r=1$ & $\gamma=2$ & $r=5$ & $r=1$ & $r=2$ & $\gamma=5$ \\
\hline \multirow{2}{*}{$1 / 4$ (CPU time) } & $0.1067 \mathrm{E}-03$ & $0.3903 \mathrm{E}-03$ & $0.8100 \mathrm{E}-02$ & $0.7776 \mathrm{E}-03$ & $0.9220 \mathrm{E}-03$ & $0.2318 \mathrm{E}-01$ \\
& $(0.2404)$ & $(0.2493)$ & $(0.2482)$ & $(0.3002)$ & $(0.3122)$ & $(0.3120)$ \\
$1 / 8$ (CPU time) & $0.9365 \mathrm{E}-05$ & $0.3437 \mathrm{E}-04$ & $0.6271 \mathrm{E}-03$ & $0.4418 \mathrm{E}-04$ & $0.6072 \mathrm{E}-04$ & $0.1272 \mathrm{E}-02$ \\
& $(1.6889)$ & $(1.7670)$ & $(2.0218)$ & $(2.1122)$ & $(2.2424)$ & $(2.5560)$ \\
$1 / 16$ (CPU time) & $0.6667 \mathrm{E}-06$ & $0.2416 \mathrm{E}-05$ & $0.4248 \mathrm{E}-04$ & $0.2218 \mathrm{E}-05$ & $0.3664 \mathrm{E}-05$ & $0.8814 \mathrm{E}-04$ \\
& $(25.9590)$ & $(27.6302)$ & $(32.5906)$ & $(31.0244)$ & $(33.4255)$ & $(40.1616)$ \\
$1 / 32$ (CPU time) & $0.4148 \mathrm{E}-07$ & $0.1523 \mathrm{E}-06$ & $0.2608 \mathrm{E}-05$ & $0.1360 \mathrm{E}-06$ & $0.2214 \mathrm{E}-06$ & $0.5416 \mathrm{E}-05$ \\
& $(367.1442)$ & $(396.0654)$ & $(455.2458)$ & $(459.1677)$ & $(495.8262)$ & $(569.1088)$ \\
\hline
\end{tabular}

Example 6.6 (quasilinear hyperbolic equation).

$$
\begin{aligned}
u_{t t}= & \left(1+u^{2}\right)\left(u_{x x}+u_{y y}\right)+\gamma u\left(u_{x}+u_{y}+u_{t}\right) \\
& +\left[\gamma e^{-t}(\sinh x \cosh y-\cosh (x+y))-1\right] e^{-t} \sinh x \cosh y \\
& -2\left(e^{-t} \sinh x \cosh y\right)^{3}, \quad 0<x, \quad y<1, \quad t>0
\end{aligned}
$$

with exact solution $u=e^{-t} \sinh x \cosh y$. The maximum absolute errors and CPU time (in seconds) are tabulated in Table 6 at $t=1$ for $\gamma=1,2$, and 5 .

The order of convergence may be obtained by using the following formula:

$$
\frac{\log \left(e_{h_{1}}\right)-\log \left(e_{h_{2}}\right)}{\log \left(h_{1}\right)-\log \left(h_{2}\right)}
$$

where $e_{h_{1}}$ and $e_{h_{2}}$ are maximum absolute errors for two uniform mesh widths $h_{1}$ and $h_{2}$, respectively. For computation of order of convergence of the proposed method, we have considered errors for last two values of $h$, that is, $h_{1}=1 / 32, h_{2}=1 / 64$ for two linear problems, and $h_{1}=1 / 16, h_{2}=1 / 32$ for all non-linear and quasilinear problems, and results are reported in Table 7. 
Table 6: Example 6.6: The maximum absolute errors (with CPU time).

\begin{tabular}{lcccccc}
\hline$h$ & \multicolumn{3}{c}{ Proposed $O\left(k^{2}+k^{2} h^{2}+h^{4}\right)$-method } & \multicolumn{3}{c}{$O\left(k^{4}+k^{2} h^{2}+h^{4}\right)$-method discussed in [7] } \\
& $\gamma=1$ & $\gamma=2$ & $\gamma=5$ & $r=1$ & $\gamma=2$ & $\gamma=5$ \\
\hline \multirow{2}{*}{$1 / 4$ (CPU time) } & $0.1286 \mathrm{E}-03$ & $0.1251 \mathrm{E}-03$ & $0.3733 \mathrm{E}-03$ & $0.6618 \mathrm{E}-03$ & $0.7715 \mathrm{E}-03$ & $0.8812 \mathrm{E}-03$ \\
& $(0.2602)$ & $(0.2607)$ & $(0.2532)$ & $(0.3288)$ & $(0.3292)$ & $(0.3166)$ \\
$1 / 8$ (CPU time) & $0.9423 \mathrm{E}-05$ & $0.8670 \mathrm{E}-05$ & $0.2271 \mathrm{E}-04$ & $0.3336 \mathrm{E}-04$ & $0.4112 \mathrm{E}-04$ & $0.5080 \mathrm{E}-04$ \\
& $(1.9640)$ & $(1.9835)$ & $(1.9870)$ & $(2.3361)$ & $(2.3410)$ & $(2.3422)$ \\
$1 / 16$ (CPU time) & $0.6313 \mathrm{E}-06$ & $0.5622 \mathrm{E}-06$ & $0.1452 \mathrm{E}-05$ & $0.2112 \mathrm{E}-05$ & $0.2446 \mathrm{E}-05$ & $0.2863 \mathrm{E}-05$ \\
& $(30.0540)$ & $(29.7286)$ & $(32.0085)$ & $(37.6212)$ & $(37.2268)$ & $(40.1020)$ \\
$1 / 32$ (CPU time) $)$ & $0.3939 \mathrm{E}-07$ & $0.3490 \mathrm{E}-07$ & $0.9018 \mathrm{E}-07$ & $0.1308 \mathrm{E}-06$ & $0.1512 \mathrm{E}-06$ & $0.1744 \mathrm{E}-06$ \\
& $(414.6224)$ & $(405.4874)$ & $(450.4474)$ & $(517.6668)$ & $(507.6543)$ & $(563.5578)$ \\
\hline
\end{tabular}

Table 7: Order of convergence.

\begin{tabular}{lcc}
\hline Example & Parameters & Order of the method \\
\hline \multirow{4}{*}{$\alpha=10, \beta=5, \eta=0.5, \gamma=1$ at $t=5$} & 4.00 \\
& $\alpha=20, \beta=10, \eta=1, \gamma=1$ at $t=5$ & 4.02 \\
& $\alpha=40, \beta=4, \eta=10, \gamma=20$ at $t=5$ & 3.99 \\
& $\alpha=50, \beta=5, \eta=0.25, \gamma=0.75$ at $t=5$ & 3.99 \\
$\alpha=10, \beta=0, \eta=5, \gamma=5$ at $t=5$ & 4.00 \\
\hline 02 & at $t=1$ & 4.00 \\
& at $t=2$ & 3.99 \\
\hline 03 & $\gamma=1$ at $t=2$ & 3.99 \\
& $\gamma=2$ at $t=2$ & 3.99 \\
\hline 04 & $\gamma=3$ at $t=2$ & 4.00 \\
\hline & $t=1$ & 3.99 \\
05 & $\gamma=1$ at $t=1$ & 4.00 \\
& $\gamma=2$ at $t=1$ & 3.99 \\
& $\gamma=5$ at $t=1$ & 4.01 \\
\hline \multirow{3}{*}{06} & $\gamma=1$ at $t=1$ & 4.00 \\
& $\gamma=2$ at $t=1$ & 4.01 \\
& $\gamma=5$ at $t=1$ & 4.00 \\
\hline
\end{tabular}

\section{Concluding Remarks}

Available numerical methods for the numerical solution of second-order quasilinear wave equations are of order four, which require 19-grid points. In this article, using the same number of grid points and five evaluations of the function $g$ (as compared to seven evaluations of the function $g$ discussed in $[6,7])$, we have derived a new stable numerical method of $O\left(k^{2}+k^{2} h^{2}+h^{4}\right)$ accuracy for the solution of quasilinear wave equation (1.1). Ultimately, we use less algebra for computation, and for a fixed parameter value $\sigma=k / h^{2}$, the proposed method behaves like a fourth-order method, which is exhibited from the computed results. The proposed numerical method is applicable to wave equation in polar coordinates, and for the damped wave equation and telegraphic equation the method is shown to be unconditionally stable. 


\section{Acknowledgments}

The authors thank the anonymous reviewer for his valuable suggestions, which substantially improved the standard of the paper. This research was supported by The University of Delhi under research Grant no. Dean (R)/R \& D/2010/1311.

\section{References}

[1] M. Ciment and S. H. Leventhal, "Higher order compact implicit schemes for the wave equation," Mathematics of Computation, vol. 29, no. 132, pp. 985-994, 1975.

[2] M. Ciment and S. H. Leventhal, "A note on the operator compact implicit method for the wave equation," Mathematics of Computation, vol. 32, no. 141, pp. 143-147, 1978.

[3] T. Schwartzkopff, C. D. Munz, and E. F. Toro, "Ader: a High-Order Approach for Linear Hyperbolic Systems in 2D," Journal of Scientific Computing, vol. 17, pp. 231-240, 2002.

[4] T. Schwartzkopff, M. Dumbser, and C. -D. Munz, "Fast high order ADER schemes for linear hyperbolic equations," Journal of Computational Physics, vol. 197, no. 2, pp. 532-539, 2004.

[5] M. Dumbser and M. Käser, "Arbitrary high order non-oscillatory finite volume schemes on unstructured meshes for linear hyperbolic systems," Journal of Computational Physics, vol. 221, no. 2, pp. 693-723, 2007.

[6] R. K. Mohanty, M. K. Jain, and K. George, "High order difference schemes for the system of two space second order nonlinear hyperbolic equations with variable coefficients," Journal of Computational and Applied Mathematics, vol. 70, no. 2, pp. 231-243, 1996.

[7] R. K. Mohanty, U. Arora, and M. K. Jain, "Linear stability analysis and fourth-order approximations at first time level for the two space dimensional mildly quasi-linear hyperbolic equations," Numerical Methods for Partial Differential Equations, vol. 17, no. 6, pp. 607-618, 2001.

[8] R. K. Mohanty and M. K. Jain, “An unconditionally stable alternating direction implicit scheme for the two space dimensional linear hyperbolic equation," Numerical Methods for Partial Differential Equations, vol. 22, no. 6, pp. 983-993, 2001.

[9] R. K. Mohanty, "An operator splitting method for an unconditionally stable difference scheme for a linear hyperbolic equation with variable coefficients in two space dimensions," Applied Mathematics and Computation, vol. 152, no. 3, pp. 799-806, 2004.

[10] M. Dehghan and A. Shokri, "A numerical method for solving the hyperbolic telegraph equation," Numerical Methods for Partial Differential Equations, vol. 24, no. 4, pp. 1080-1093, 2008.

[11] M. Dehghan and A. Mohebbi, "High order implicit collocation method for the solution of twodimensional linear hyperbolic equation," Numerical Methods for Partial Differential Equations, vol. 25, no. 1, pp. 232-243, 2009.

[12] M. Dehghan and A. Shokri, "A meshless method for numerical solution of a linear hyperbolic equation with variable coefficients in two space dimensions," Numerical Methods for Partial Differential Equations, vol. 25, no. 2, pp. 494-506, 2009.

[13] R. K. Mohanty, "New unconditionally stable difference schemes for the solution of multi-dimensional telegraphic equations," International Journal of Computer Mathematics, vol. 86, no. 12, pp. 2061-2071, 2009.

[14] J. Liu and K. Tang, "A new unconditionally stable ADI compact scheme for the two-spacedimensional linear hyperbolic equation," International Journal of Computer Mathematics, vol. 87, no. 10, pp. 2259-2267, 2010.

[15] Y. Y. Hu and H. W. Liu, "An unconditionally stable spline difference scheme for solving the second 2D linear hyperbolic equation," in Proceedings of the 2nd International Conference on Computer Modeling and Simulation (ICCMS '10), vol. 4, pp. 375-378, 2010.

[16] S. Karaa, "Unconditionally stable ADI scheme of higher-order for linear hyperbolic equations," International Journal of Computer Mathematics, vol. 87, no. 13, pp. 3030-3038, 2010.

[17] R. K. Mohanty and S. Singh, "High accuracy Numerov type discretization for the solution of onespace dimensional non-linear wave equations with variable coefficients," Journal of Advanced Research in Computer Science, vol. 3, pp. 53-66, 2011.

[18] M. Lees, "Alternating direction methods for hyperbolic differential equations," vol. 10, pp. 610-616, 1962. 
[19] A. R. Gourlay and A. R. Mitchell, "A classification of split difference methods for hyperbolic equations in several space dimensions," SIAM Journal on Numerical Analysis, vol. 6, pp. 62-71, 1969.

[20] C. T. Kelly, Iterative Methods for Linear and Non-Linear Equations, SIAM Publications, Philadelphia, Pa, USA, 1995.

[21] Y. Saad, Iterative Methods for Sparse Linear Systems, Society for Industrial and Applied Mathematics, Philadelphia, Pa, USA, 2nd edition, 2003.

[22] L. A. Hageman and D. M. Young, Applied Iterative Methods, Dover Publications Inc., Mineola, NY, USA, 2004.

[23] R. K. Mohanty, "Stability interval for explicit difference schemes for multi-dimensional second-order hyperbolic equations with significant first-order space derivative terms," Applied Mathematics and Computation, vol. 190, no. 2, pp. 1683-1690, 2007.

[24] M. M. Chawla, "Superstable two-step methods for the numerical integration of general second order initial value problems," Journal of Computational and Applied Mathematics, vol. 12, pp. 217-220, 1985. 


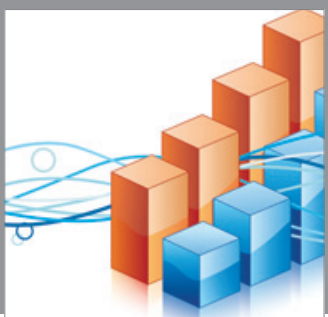

Advances in

Operations Research

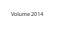

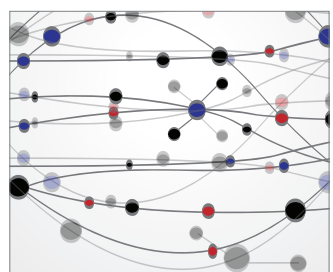

\section{The Scientific} World Journal
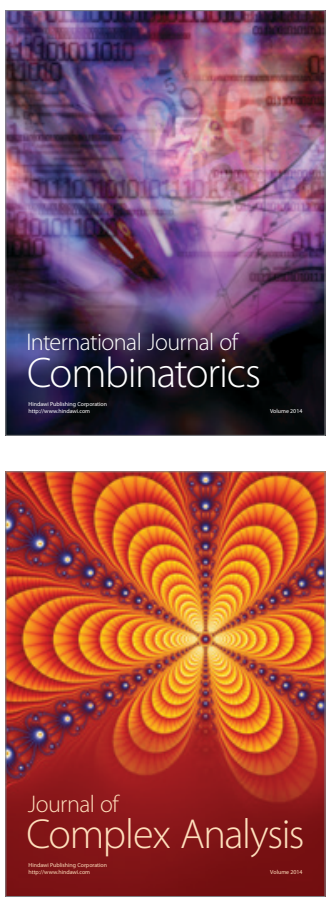

International Journal of

Mathematics and

Mathematical

Sciences
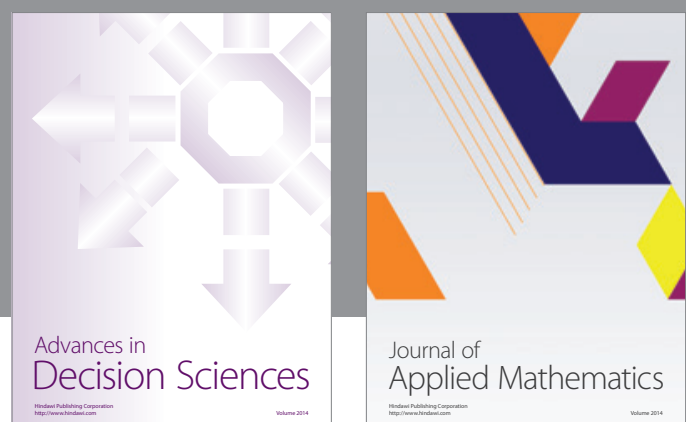

Journal of

Applied Mathematics
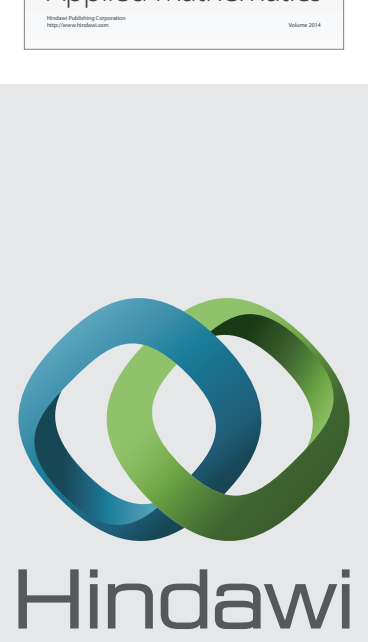

Submit your manuscripts at http://www.hindawi.com
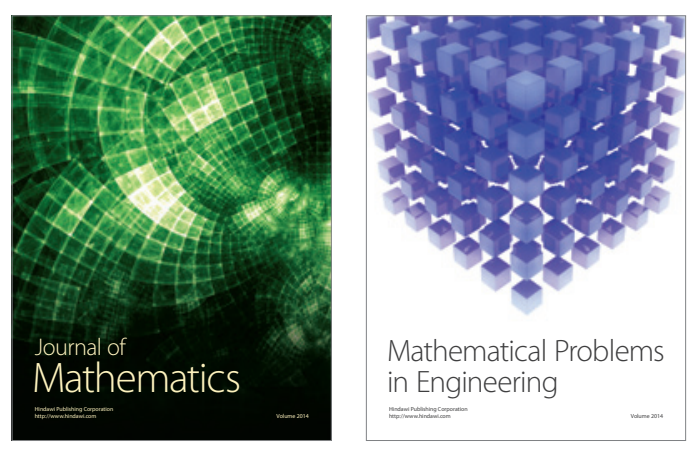

Mathematical Problems in Engineering
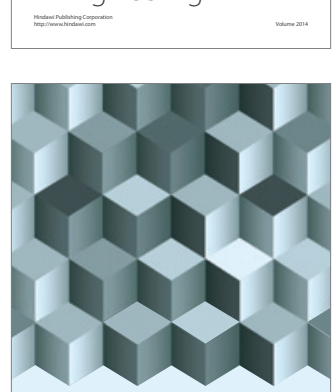

Journal of

Function Spaces
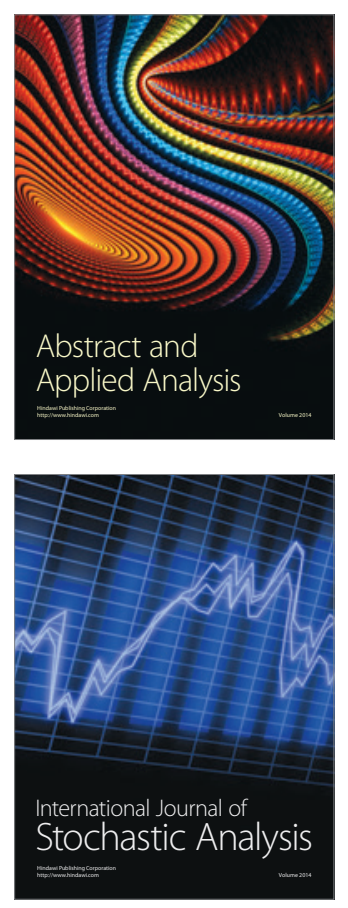

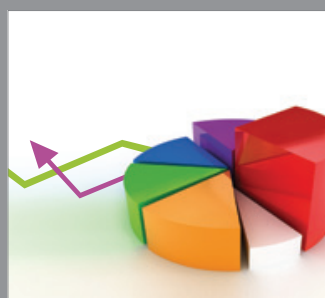

ournal of

Probability and Statistics

Promensencen
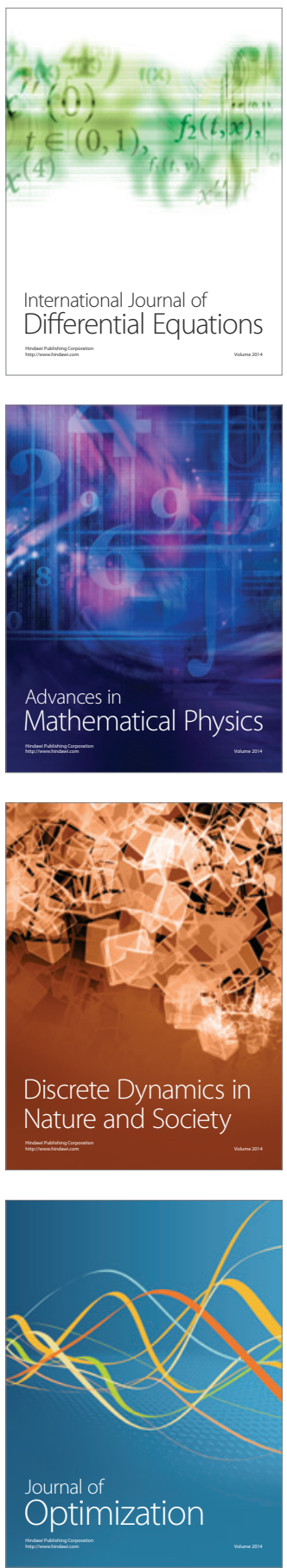